\title{
Rachel Silvera, Un quart en moins. Des femmes se battent pour en finir avec les inégalités de salaire
}

Paris, La Découverte, coll. « Cahiers libres », 2014

\section{Vincent-Arnaud Chappe}

\section{(2) OpenEdition}

\section{Journals}

Édition électronique

URL : http://journals.openedition.org/travailemploi/6741

DOI : $10.4000 /$ travailemploi.6741

ISSN : 1775-416X

Éditeur

DARES - Ministère du Travail

Édition imprimée

Date de publication : 1 juillet 2015

Pagination : 80-81

ISSN : 0224-4365

\section{Référence électronique}

Vincent-Arnaud Chappe, «Rachel Silvera, Un quart en moins. Des femmes se battent pour en finir avec les inégalités de salaire », Travail et Emploi [En ligne], 143 | juillet-septembre 2015, mis en ligne le 01 juillet 2015, consulté le 10 décembre 2020. URL : http://journals.openedition.org/travailemploi/6741 ; DOI : https://doi.org/10.4000/travailemploi.6741

Ce document a été généré automatiquement le 10 décembre 2020.

(c) Direction de l'animation de la recherche, des études et des statistiques (Dares) 


\section{Rachel Silvera, Un quart en moins. Des femmes se battent pour en finir avec les inégalités de salaire}

Paris, La Découverte, coll. « Cahiers libres », 2014

\section{Vincent-Arnaud Chappe}

\section{RÉFÉRENCE}

Rachel Silvera, Un quart en moins. Des femmes se battent pour en finir avec les inégalités de salaire, Paris, La Découverte, coll. «Cahiers libres », 2014, 224 p.

1 Pourquoi en France, presque soixante-dix ans après que le préambule de la Constitution de la IV ${ }^{e}$ République a institué le principe d'égalité entre les sexes, les femmes salariées continuent-elles à toucher en moyenne "un quart en moins" de salaire que leurs homologues masculins ? Comment expliquer la persistance d'un écart si important et la lenteur de son érosion malgré l'édiction d'une législation antidiscriminatoire foisonnante? Et quels sont les leviers susceptibles de modifier cet état de fait et de s'approcher enfin d'une égalité réelle? C'est sur ces problématiques à la fois sociologiques, économiques et politiques que l'économiste Rachel Silvera, spécialiste des questions d'égalité professionnelle, se penche dans Un quart en moins. Des femmes se battent pour en finir avec les inégalités de salaire, publié à La Découverte en 2014.

Préfacé par Michelle Perrot, le livre est composé de trois parties de deux chapitres, chacune adoptant un point de vue spécifique sur la question. La première partie - « Le spectre du salaire d'appoint »- développe une approche historique. L'auteure propose une stimulante synthèse des travaux d'histoire s'étant intéressés au "salaire féminin " en France tout au long du $\mathrm{xx}^{\mathrm{e}}$ siècle. Elle analyse les arguments patronaux - mais également syndicaux ou théorisés par les économistes classiques - qui ont longtemps justifié la sous-valorisation salariale du travail féminin, puis relate la « longue bataille » des militantes féministes et syndicales qui a abouti à la progressive 
désinstitutionnalisation de cette inégalité, à défaut de sa résorption dans les faits. Selon R. Silvera, l'écart salarial contemporain entre femmes et hommes trouve sa source dans l'historique dévalorisation du travail des femmes, censées n'apporter qu'un «salaire d'appoint». La mission de faire vivre le ménage était dévolue à l'homme "chef de famille ", qu'il soit le mari ou le père. Dans cette perspective, la situation des femmes vivant seules était laissée de côté, au profit de la norme de la femme mariée pouvant compter sur le salaire de son conjoint. Les mobilisations syndicales et les bouleversements économiques liés aux guerres ont progressivement légitimé le travail féminin, mais sans éliminer pour autant totalement l'idée du " salaire d'appoint », dont témoigne notamment la forte féminisation de l'emploi à temps partiel.

3 La deuxième partie du livre - «Elles se battent aujourd'hui »- s'écarte de l'approche historique, pour s'intéresser aux processus de discrimination professionnelle et aux mobilisations judiciaires de femmes ayant voulu y mettre fin. R. Silvera y livre un certain nombre de témoignages de femmes ayant connu des carrières entravées. Ces portraits conduisent le lecteur à appréhender la complexité des mécanismes inégalitaires, et montrent que les discriminations que subissent les femmes doivent être abordées de manière diachronique, la comparaison synchronique ceteris paribus ne permettant pas de saisir le rôle des dynamiques temporelles dans le creusement des inégalités. L'auteure met notamment en avant "l'impact des filières sexuées" (administrative, médicosociale, etc.) en matière d'inégal accès à la carrière, et le "soupçon de la maternité " qui pèse sur toutes les femmes et leur évolution professionnelle. Face à ces injustices, les salariées ne sont néanmoins pas totalement démunies: celles qui ont relaté leur expérience ont en commun d'avoir saisi les tribunaux pour demander la réparation du préjudice subi. S'appuyant sur le droit de la non-discrimination et une méthode de preuve mise en place à l'origine pour lutter contre les discriminations syndicales - la méthode « $\operatorname{Clerc}^{1}$ »- elles ont pu, au bout d'un long parcours souvent semé d'embûches, faire valoir le préjudice subi et le manque à gagner salarial accumulé sur des années et se répercutant sur leurs retraites.

4 La troisième partie - «À travail de valeur égale, salaire égal !» - dégage une troisième piste explicative des inégalités salariales: les "emplois à prédominance féminine ", c'est-à-dire ceux où les femmes sont très largement surreprésentées ${ }^{2}$, sont également les emplois les moins bien valorisés salarialement dans les conventions collectives ou au sein des entreprises. Deux facteurs concourent à expliquer le phénomène : emplois relevant majoritairement des «fonctions support» (ressources humaines, administration, communication, etc.), considérées comme moins essentielles que les fonctions « cœur de métier ", ils ouvrent à moins de possibilités d'évolution de carrière et sont historiquement moins valorisés que ceux à prédominance masculine. De plus, ils requièrent souvent un ensemble de compétences peu formalisables (telles que les " compétences relationnelles ») et peu reconnues car réputées "innées » chez les femmes. S'appuyant à nouveau sur des témoignages de femmes, R. Silvera dévoile ainsi à la fois l'importance des compétences et responsabilités exercées dans certains emplois faiblement valorisés (auxiliaire de vie, agent d'entretien, etc.), et les luttes menées au sein de certaines professions féminisées - comme les infirmières ou les sages-femmes - pour une plus grande reconnaissance de leur travail.

5 Pédagogique et très agréable à lire, l'ouvrage a tout d'abord le grand mérite de donner la parole à des femmes discriminées et ayant lutté pour leurs droits. Ces portraits, sous forme de témoignages, nous plongent directement dans le vécu complexe de la 
discrimination et de l'invisibilisation du travail des femmes. À partir de ces récits, l'auteure montre de façon tout à fait convaincante la nécessité de ne pas s'en tenir à une analyse «toutes choses égales par ailleurs » de la discrimination salariale : celle-ci doit au contraire être appréhendée de façon dynamique, c'est-à-dire dans l'évolution de la carrière, mais également à travers le cloisonnement et la ségrégation horizontale des métiers féminins. Au-delà du constat et de l'explication, le propos de R. Silvera est orienté vers l'action dans une perspective que Michelle Perrot qualifie dans la préface d'« optimiste » (p. 8) : cet optimisme s'exprime d'abord dans la présentation de combats judiciaires victorieux, démonstration par l'exemple d'une certaine efficacité de l'arme du droit. Mais l'ouvrage ne se limite pas à ces cas singuliers : il développe en sus un ensemble de pistes de réformes politiques qui pourraient rendre le droit à l'égalité réelle encore plus effectif. L'auteure aborde ainsi la question de l'action judiciaire de groupe - sur le modèle américain de la class action -, de l'enrichissement des indicateurs de suivi des inégalités au sein des entreprises, et de la nécessité de procéder à des études systématiques sur l'impact sexué des classifications professionnelles, à l'instar de ce que prévoit la loi sur l'équité salariale au Québec.

Ces différentes mesures ont en commun de faciliter le passage d'une approche au cas par cas de la discrimination, telle qu'elle se manifeste dans les contentieux individuels portés devant les tribunaux, à une approche collective qui prend en compte le caractère organisationnel et systémique des inégalités entre femmes et hommes.

Quelques critiques peuvent être formulées. Elles sont d'abord d'ordre méthodologique : on ne sait pas grand-chose de la démarche employée, de la façon dont a été constitué l'échantillon de femmes interrogées ou des modalités de recueil des témoignages. De même, la nature des récits livrés tout au long de l'ouvrage n'apparaît pas clairement: s'ils sont rédigés à la première personne du singulier, le style suggère une réécriture par l'auteure qui n'est jamais précisée.

8 Plus fondamentalement, on peut regretter que l'auteure ne s'appuie que très peu sur les apports de la sociologie du droit pour analyser les parcours judiciaires des victimes de discrimination. Ce sous-champ de la sociologie s'est pourtant largement développé en France depuis une dizaine d'années, un certain nombre de travaux concernant directement la question du recours judiciaire face aux discriminations. L'exploitation de leurs résultats aurait pu permettre d'analyser plus finement les obstacles et contraintes que rencontrent les victimes de discrimination dans leur périple judiciaire.

Ces limites doivent néanmoins être grandement relativisées au regard de l'objectif du livre: Un quart en moins est d'abord un ouvrage pédagogique et militant d'une grande richesse. Instructif à de nombreux égards, proposant une excellente synthèse des mécanismes de l'inégalité professionnelle entre femmes et hommes, il offre également des pistes de réforme à la fois ambitieuses et réalistes qui permettront, on peut l'espérer, d'en finir à terme avec une injustice longtemps considérée comme une fatalité. 


\section{NOTES}

1. La méthode «Clerc »-du nom de François Clerc, syndicaliste CGT à Peugeot PSA - est une méthode permettant d'objectiver la discrimination, de la représenter graphiquement et de la quantifier à partir du tracé sur un même graphique de la courbe d'évolution salariale du plaignant et de celle d'un groupe d'homologues (entrés à la même période dans l'entreprise sur le même poste) non concernés par la discrimination.

2. Par exemple les infirmières ou les secrétaires.

\section{AUTEURS}

\section{VINCENT-ARNAUD CHAPPE}

Centre de sociologie de l'innovation - Institut interdisciplinaire de l'innovation (CSI-i3, CNRS,

Mines ParisTech, UMR 9217) 